\title{
Diagnosis of asbestosis by needle lung biopsy
}

\author{
M. WALTON ANDT. SKEOCH \\ From Poole Hospital and Central Clinical Laboratories, Middlesbrough
}

Nine patients from Teesside who had asbestosis are briefly described. In seven of them a needle biopsy was made to confirm the diagnosis. The advantages of the procedure, using the Jack needle (Smith, 1964a), are discussed.

We are recognizing asbestosis with increasing frequency on Teesside. During the past year those working in this area in industry in which asbestos is used have been made aware of the possible serious consequences of dust inhalation by the publicity given to a coroner's report (Northern Daily Mail, 1965). This report was on the pathological findings in an asbestos worker who died from peritoneal mesothelioma. The patient was a member of a contractor's 'lagging team' and after his death chest radiographs were taken of the workers of his gang. From this group of men further cases of asbestosis were diagnesed and workers exposed to asbestos in other industries came forward for examination.

The difficulties of diagnosing asbestosis are well recognized and referred to, particularly in the paper by Williams and Hugh-Jones (1960a), in which they describe lung function changes in 21 certified cases and 10 patients with exposure to asbestos but no definite signs. Loss of lung function is still the sheet anchor of diagnostic criteria, but the functional changes, e.g., lowered transfer factor, reduced inspiration capacity, hyperventilation with arterial desaturation on exertion, together with no evidence of airway obstruction, are common to other interstitial fibroses. These lung function changes precede the characteristic radiographic changes. In a further paper by Williams and Hugh-Jones (1960b) the difficulties in radiological diagnosis are discussed. It is stated that the appearances are quite unspecific except in advanced cases.

A significant advance in diagnosis has been made by lung biopsy, using the Jack needle, which has enabled a precise histological diagnosis to be made.

The reaction of the lungs and pleura to asbestos fibre is widespread fibrosis, involving particularly the lower parts of the lung. The pleural space may be obliterated, and when this occurs the complication of pneumothorax, which commonly follows a needle biopsy, is avoided.

The Jack needle was described by Smith i 1964 and is essentially a punch. In a letter to the British Medical Journal (1964) shortly after the original paper in Thorax, Smith reported a fatality from uncontrolled haemorrhage in a case oळo sarcoidosis. This followed a punch at $5 \mathrm{~cm}$. depth In a personal communication (1968) Smith states that he has largely given up the needle and, in any case, would only use it in an operating theatre or at least where full resuscitation facilities are available. We have performed over 200 needle lung biopsies without fatality, but must stress tha $\overrightarrow{\widehat{\Phi}}$ the punch is used only in a hospital where ful thoracic surgical facilities are immediately avai able. Such a precaution seems to us to be common-sense attitude to a potentially hazardoust investigation. We also limit the depth of the punce to 1 to $2 \mathrm{~cm}$. beyond the pleura.

Premedication with an oral barbiturate and atropine is advisable. The site of puncture is detero mined by careful study of the radiographs ; 6-1 $\mathrm{ml}$. of $1 \%$ lignocaine is infiltrated as far as the parietal pleura and massaged. A second puncture is made with a separate trocar which avoid? scalpel blade cutting. Having checked that the punch should open $5-6 \mathrm{~mm}$. in the open position the domed nut is tightened against the adjusting nut. This ensures that the inner rod and point cap be held immobile while the outer cutting cannulfis is rotated against the anvil in the closed position? The closed needle is sharply advanced about $1-2 \mathrm{~cm}$. into the lung beyond the pleura during held inspiration. The punch is then opened and the whole assembly is withdrawn slightly to ensure trapping a portion of lung on the snag. The punct is then sharply and firmly closed. Whilst main $\frac{P}{\mathbb{D}}$ taining a firm pressure in this position, the inne? rod is held immobile and the outer needle is rota ted through an arc of $90^{\circ}$ in an oscillatory manner 
The needle is then quickly withdrawn, being firmly held meantime in the closed position. Speed and firmness of action are essential in the successful use of the needle. It should not be necessary for the needle to remain in the lung for more than 5-6 seconds. The amount of tissue removed with the needle is in most cases very small, but even pieces of $1 \times 0.5 \mathrm{~mm}$., if carefully handled, can be satisfactorily processed. We advise that the fresh tissue be lightly wrapped in a cigarette paper and, without being allowed to dry, dropped into formol saline. The tissue, while still wrapped, is fully processed and on removal from the vacuum wax bath the paper is opened with two pairs of warmed forceps and the impregnated tissue is blocked out in the usual way. By processing in this way, handling of the tissue is reduced to a minimum and crush distortion does not occur.

\section{CASE HISTORIES}

CASE 1 A hospital maintenance engineer, born in 1902, first complained in 1958 of shortness of breath and tiredness on exertion. His chest radiograph at that time showed some loss of translucency on the right side with some shadowing near the septum in the right mid-zone, but these changes were minimal.

He remained at work in spite of increasing dyspnoea until June 1965, when he was off work and was regarded as having some functional overlay. The chest radiograph then showed a further loss of translucency in the right upper zone and right mid-zone peripherally. He returned to work until November 1966, when he was referred to hospital with orthopnoea, chest pain, and haemoptysis. A loud pleural rub was heard on auscultation over the right lower chest. Asbestos bodies were found in the sputum and the chest radiograph (Fig. 1) showed a loss of the right costophrenic angle with elevation of the diaphragm. A needle biopsy specimen from the right lower lobe measured $2 \times 1 \mathrm{~mm}$. and consisted of an area of fibrosis with deep carbon pigmentation. The alveolar septa were thickened, giving rise to a 'fibrosing alveolitis' in which asbestos bodies were present, some of which showed fragmentation.

CASE 2 A chemical works labourer, born in 1912, began to complain of right chest pain in 1963. It was pleural in character and was severe enough to require his admission to hospital. At that time a diagnosis of pneumonia was made and he was treated with antibiotics and made a good recovery. There is no initial chest film available now.

He was referred to us in April 1966 with a recurrence of right-sided pleurisy, and a chest radiograph then showed a large, homogeneous opacity laterally in the right lung with small calcified areas throughout the remainder of both lung fields (Fig. 2). On physical examination there were signs of consolidation over

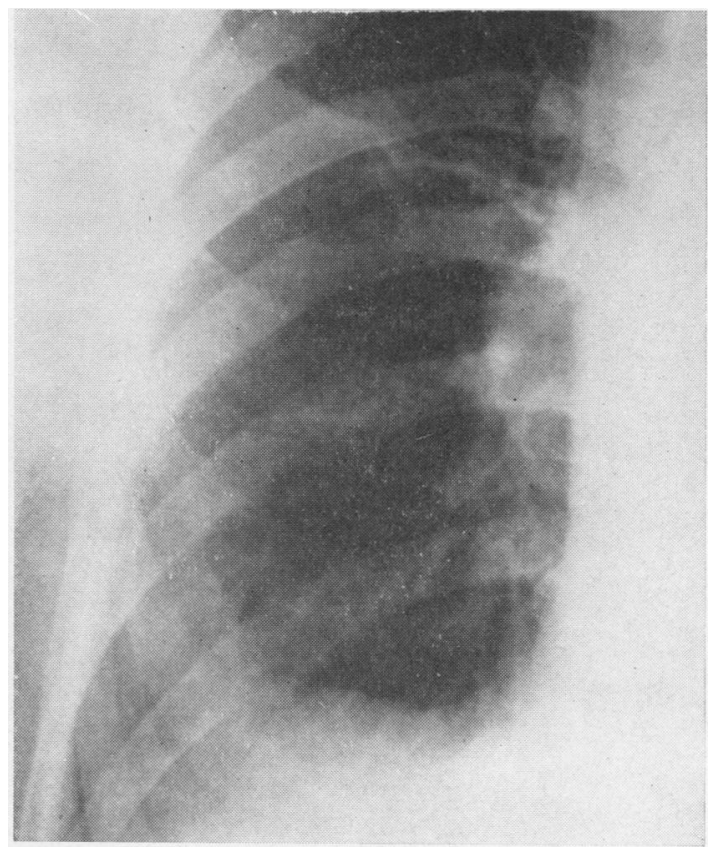

FIG. 1. Case 1. Postero-anterior radiograph showing loss of translucency on the right side.

the base of the right upper lobe posteriorly, and on auscultation crackling crepitations were heard extending forward from this area. At that time tuberculosis seemed to be the most likely diagnosis, but repeated sputum examinations for acid- and alcohol-fast bacilli and cultures for Myco. tuberculosis were negative. Asbestos bodies were not demonstrated in the sputum.

Bronchoscopy showed right-sided bronchial distortion and the aspirated mucopus contained no malignant cells. A bronchogram confirmed extensive bronchial distortion in the right upper lobe. A needle biopsy from the right upper lobe measured $1 \times 0.75$ $\mathrm{mm}$. and consisted of an area of densely fibrosed tissue with only a few alveolar septa which were thickened by 'fibrosing alveolitis'. Numerous asbestos bodies were present, some showing fragmentation (Fig. 3). On broad-spectrum antibiotics the lung opacity largely cleared, and Fig. 4 shows the present position with the small areas of calcification unchanged.

CASE 3 This patient, born in 1908, developed hoarseness of voice in June 1965 and was referred to the ear, nose, and throat department, where his left vocal cord was found to be paralysed. He had been a lagger until 1956, and smoked 30 to 40 cigarettes a day. His chest radiograph showed extensive opacities in both bases with enlargement of the left root shadow. Sputum examination on three occasions revealed asbestos bodies. Bronchoscopy showed some 


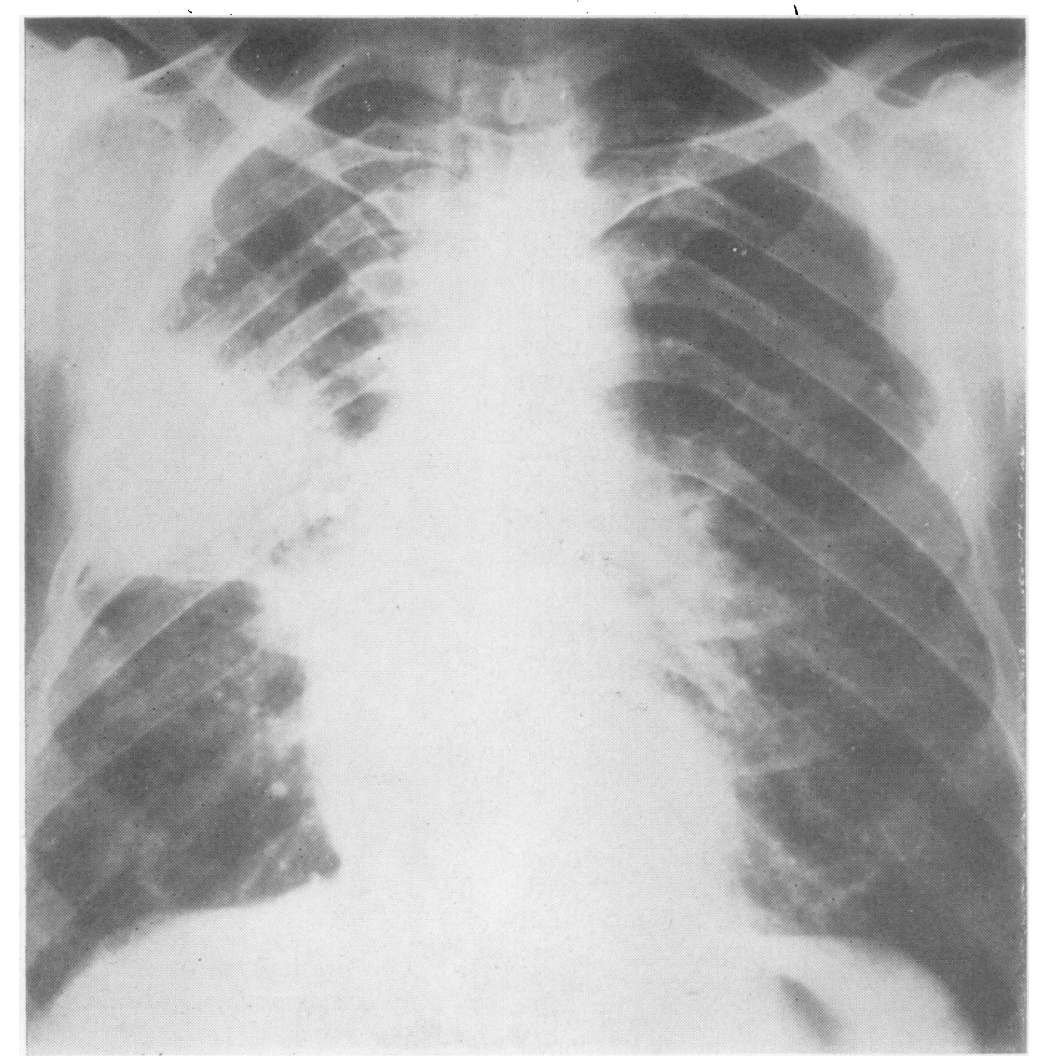

FIG. 2. Case 2. Postero-anterior radiograph showing a large homogeneous opacity laterally in the right lung, with small calcified areas throughout the remainder of the lung fields.

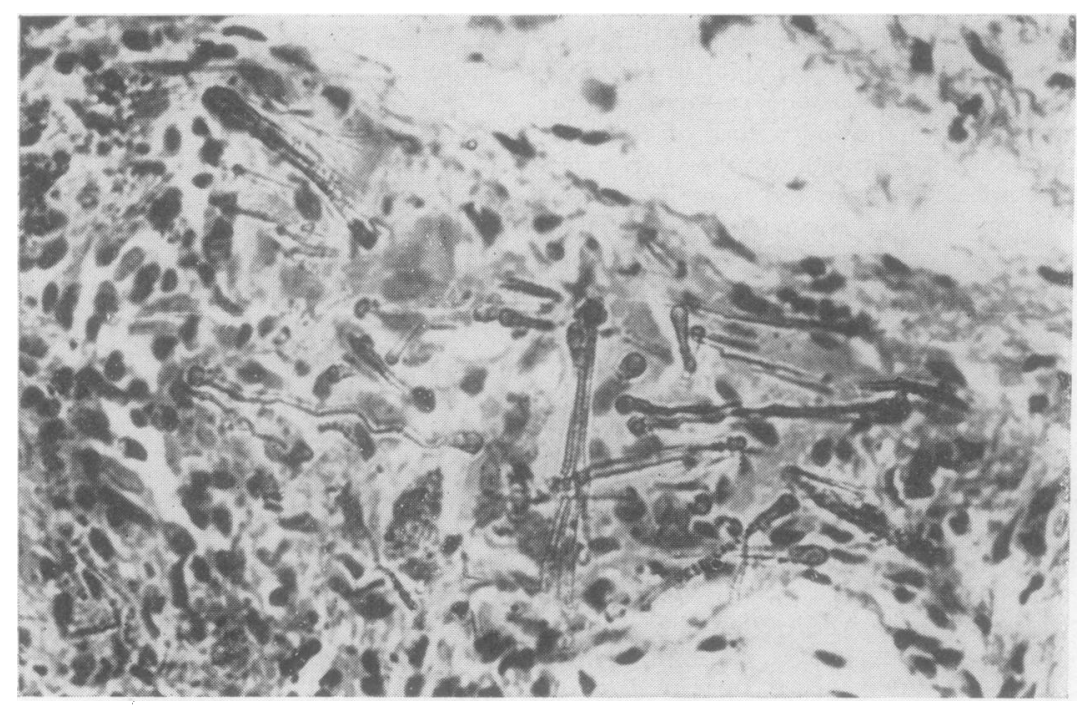

FIG. 3. Case 2. Needle biopsy showing fibrosis with numerous asbestos bodies, some of which are fragmented. 


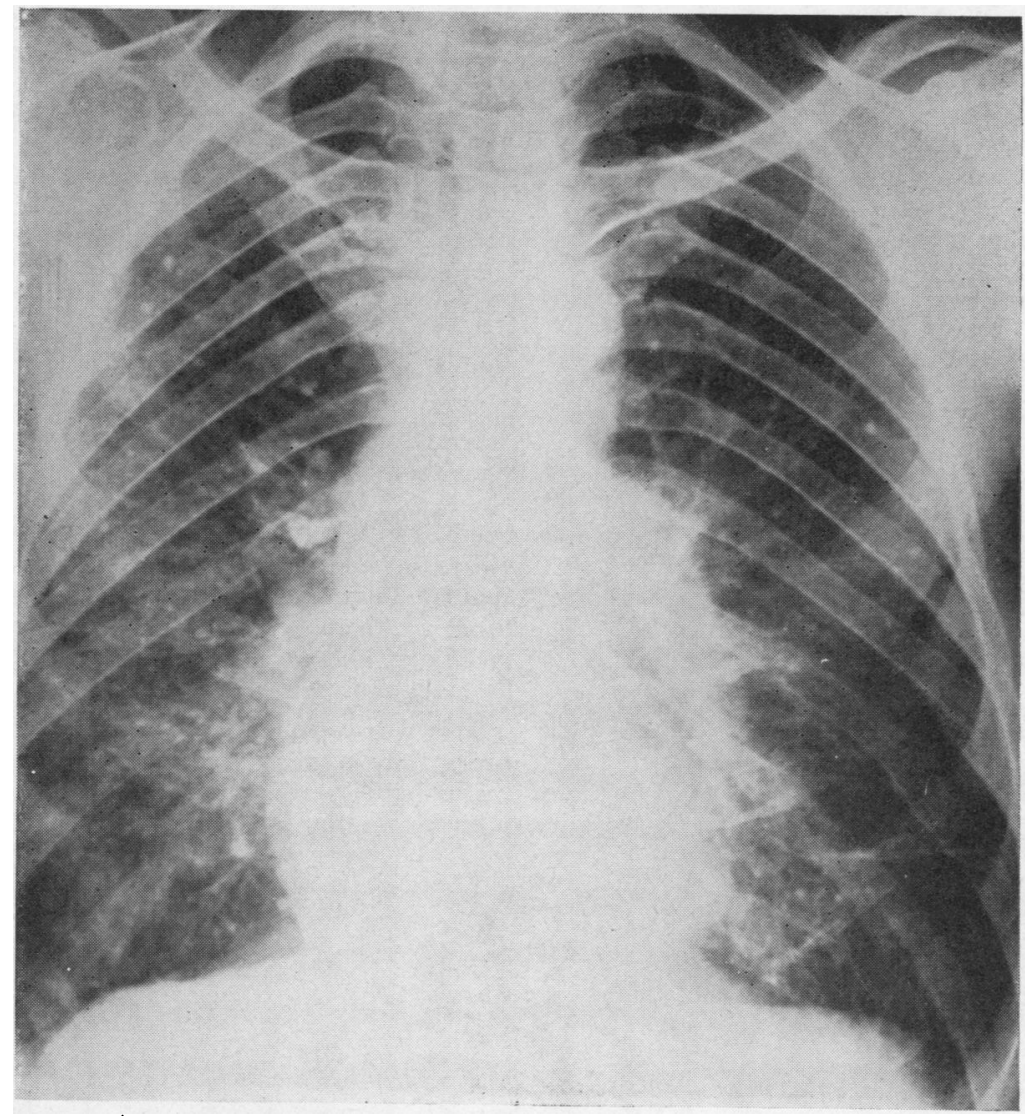

FIG. 4. Case 2. Radiograph after resolution of the pneumonitis.

narrowing of the left upper lobe orifice, and a bronchogram confirmed this finding. A needle biopsy showed pulmonary fibrosis with asbestos bodies.

He died on 18 March 1966 and at necropsy a large bronchial cancer was seen arising from the left upper lobe stem and extensive fibrosis and distortion were seen in both lower lobes.

Figure 5 shows a whole-lung section stained with prussian blue to show the take-up of stain in the area involved in the asbestotic change. The bronchial cancer is also seen.

CASE 4 This man, born in 1931, had been a lagger for 15 years and was referred for examination because of increasing exertional dyspnoea.

He smoked 20 to 30 cigarettes a day. The chest radiograph showed bilateral basal opacities with pleural thickening. Bronchoscopy showed no abnormality. Asbestos bodies were not seen in the sputum on three consecutive examinations. The bronchogram (Fig. 6) showed distortion and displacement of both the lower lobe bronchi, which had the appearance of a 'flowing moustache'. A needle biopsy showed parenchymal fibrosis in relation to fragmented asbestos bodies.

CASE 5 This man, born in 1925, had worked as a lagger for 15 years and was referred complaining of shortness of breath. The chest radiograph showed bilateral basal fibrosis. Sputum examinations on three occasions showed asbestos bodies.

Bronchoscopy showed no abnormality, but the bronchogram confirmed distortion and displacement of the lower lobe bronchi on both sides. A needle biopsy revealed pulmonary fibrosis in relation to asbestos bodies.

CASE 6 This man, born in 1918, had been a lagger for 32 years and was referred for investigation of increasing shortness of breath.

The chest radiograph showed obvious basal fibrosis with a shaggy heart shadow. Sputum examinations for asbestos bodies were negative on three occasions. Bronchoscopy showed no abnormality, but a bronchogram revealed marked basal ectatic distortion and 


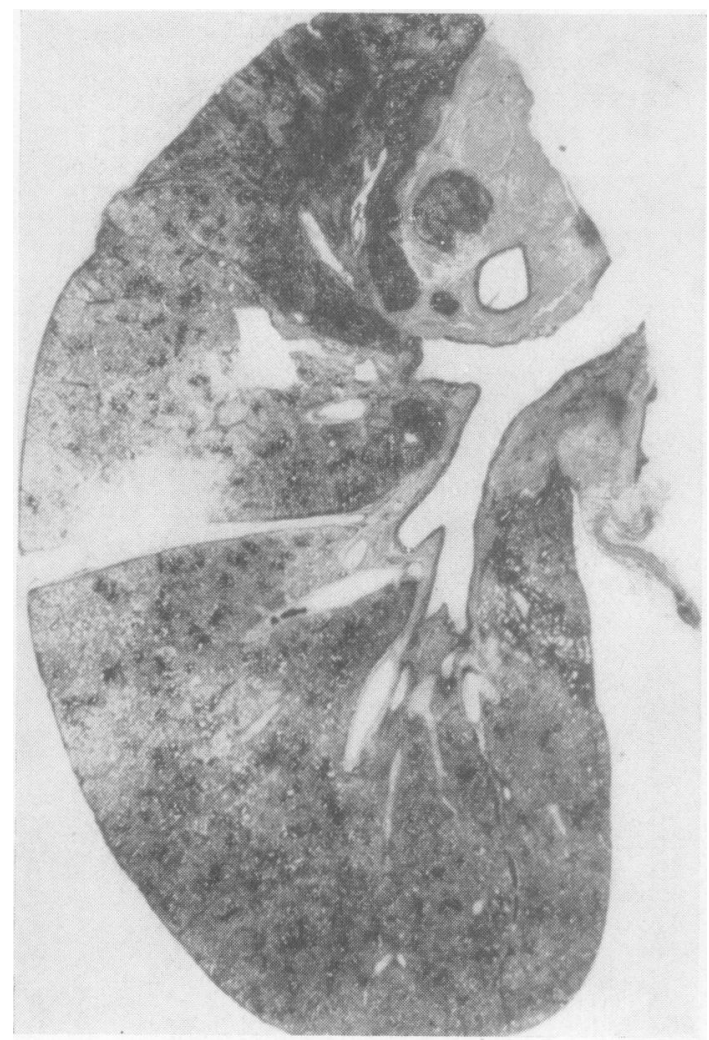

FIG. 5. Case 3. Whole-lung section stained with prussian blue to show the area involved in the asbestotic change. The bronchial cancer is also seen.

displacement of the basal bronchi. A needle biopsy showed pulmonary fibrosis with asbestos bodies.

CASE 7 This man, born in 1902, attended the Chest Clinic for many years under the label of chronic bronchitis. His main complaint was dyspnoea on exertion. His chest radiograph (Fig. 7) showed increased basal shadowing with pleural thickening and calcified pleural plaques running laterally down the chest wall and across the diaphragm.

His industrial history, which included a report from his works Industrial Medical Officer, was that from 1921 onwards he had been employed as a lagger and was eventually transferred to canteen duties when shortness of breath supervened. Sputum examinations on three occasions were negative for asbestos bodies. Bronchoscopy showed no abnormality. A bronchogram showed distortion and displacement of the lower lobe bronchi in the typical moustache pattern. A needle biopsy showed pulmonary fibrosis in relation to asbestos bodies.
CASE 8 This lady, born in 1927, was a packer in a chemical factory dealing with asbestos, and was referred because of chest pain. She had suffered from pleurisy and pneumonia in 1953, which had left her short of breath on exertion with persistent right lower chest pain. A chest radiograph showed an o opacity in the right lower chest. Her Mantoux was $\vec{\circ}$ strongly positive, but repeated attempts at chest $\vec{\overrightarrow{ }}$ aspiration failed. Bronchoscopy showed no abnor- $\vec{\omega}$ mality. A bronchogram showed poor filling of the right lower lobe bronchi. She was regarded as tuber- $\vec{x}$ culous and a trial was made of anti-tuberculosis iv drugs.

She deteriorated with persistent pain, and so a $f$ right thcracotomy was performed, when a dense hard or pleura 0.5 in. thick was found, the lung and pleura 을 being firmly fused. Histology of the surgical pleural $\rightarrow$ biopsy revealed a background having a densely $\mathcal{C}$ cellular sarcomatous appearance with epithelial-type $\mathbb{D}$ tubules. There was sharp demarcation between the $\mathbb{D}$ two types and areas were somewhat similar to those in malignant synovioma.

This was considered to be a mesothelioma, but unfortunately a necropsy was not possible to exclude the possibility of there being another source of the primary tumour.

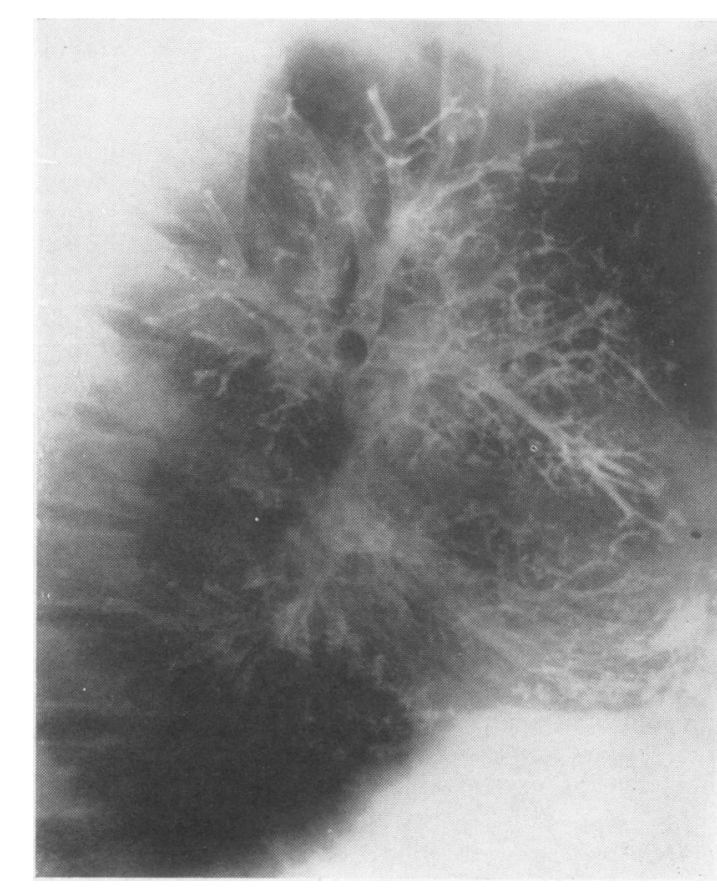

FIG. 6. Case 4. Bronchogram showing distortion and displacement of the lower lobe bronchi, likened to a flowing moustache. 


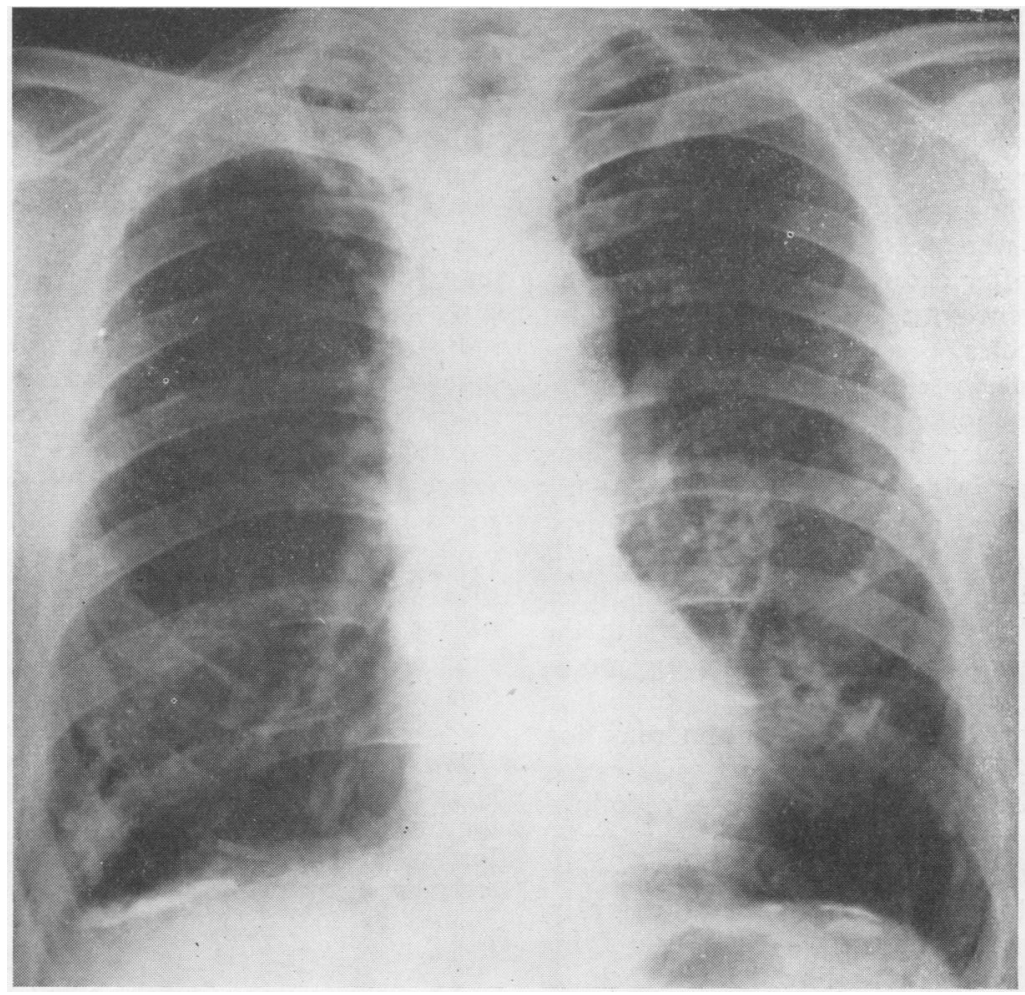

FIG. 7. Case 7. Posterc-anterior radiograph showing pleural thickening and calcified plaques.

CASE 9 This insulating engineer, born in 1917, had a 25 years' history of exposure to asbestos dust and was admitted to hospital in May 1965 with severe abdominal pain. Ascites was present and cytological examination of the fluid revealed malignant cells thought to be adenocarcinoma. His abdomen was aspirated on several occasions, but he died cachectic on 2 October 1965.

Necropsy showed a large amount of blood-stained fluid in the abdomen and extensive, nodular, soft, pale, neoplastic tissue covering the abdominal viscera and grossly matting the intestines. The outer surface of the stomach was largely covered by a flat grey mat. No primary tumour was found in the stomach, intestines, kidneys, pancreas, bladder, prostate or testes, and there were no secondary deposits in the substance of the liver, adrenals or other organs. Sections of the abdominal neoplasm showed a floridly mixed pattern of densely cellular sarcomatous appearance with easily found mitoses and papillary mucoid areas. Asbestos bodies were easily found in the lung section and there were also scattered papillary epithelial foci with asbestos bodies nearby. This was labelled as peritoneal mesothelioma. It is of interest to note that no asbestos bodies were seen in the peritoneal tumour.

\section{DISCUSSION}

We reproduce here the chest radiographs of cases 1 and 2 (Figs 1 and 2) and the lung biopsy histology of case 2 only (Fig. 3). These radiographs are atypical in that the classical distribution of change in asbestosis is not present. In case 1 the first radiological abnormality was some loss of translucency on the right side. This subsequently progressed to involve the loss of the right costo-phrenic angle and, after a lapse of eight years, both lower zones showed distortion of the lung architecture and pleural thickening. The clue to the development of asbestosis in case 1 was given when he described his work from 1958 to include the dismantling of lagged water tanks from the confined roof spaces of the wards of an E.M.S. type hutted hospital. This work was performed in addition to his normal maintenance engineering duties. The installation of a central boiler plant at the hospital had rendered redundant the individual hot water system of each ward. He had to gain access to the unventilated roof spaces and tear off the lagging 
before he was able to dismantle the water system pipes.

In case 2 the presenting lesion was a recurring pneumonic consolidation in the right upper lobe. This was due to gross bronchial deformities confirmed on bronchography when the displacement and sacculation were seen. The needle biopsy of this area taken after careful radiological planning showed the parenchymal changes in association with asbestos bodies.

All the other cases fell into the accepted grouping of asbestosis: cases 4,5 , and 6, pulmonary asbestosis with lower zone fibrosis; case 3, lung cancer with asbestosis ; case 7 , pleural calcification ; case 8 , pleural mesothelioma ; case 9 , peritoneal mesothelioma.

We have noticed in bronchography of these cases the bronchial distortion associated with the fibrosis and have likened it to the flowing moustache (Fig. 6). These areas of bronchial distortion and displacement are prone to sputum retention and consequent infective episodes.

The nine cases diagnosed on Teesside following widespread publicity about the danger of inhaling asbestos do not represent the prevalence of asbestosis in this industrial complex. Some of the cases diagnosed by means of lung biopsy were already attending hospital clinics because of chest symptoms, but the underlying pathology had not been recognized prior to needle biopsy.

The increased attention paid to the possibility of asbestosis will discover the established cases, and continued attention to the radiological aspects and the respiratory function of 'at risk' personnel will lead to earlier recognition. Up to now stress has been laid on the physiological changes in lung function as a diagnostic criterion for asbestosis
(Hunt, 1965 ; Thomson, Pelzer, and Smither, 1965). The demonstration of asbestos bodies lying in the lung parenchyma in association with areas with fibrosis is accepted as proof of the establishment of the disease.

We had failed to diagnose asbestosis on Teesside because of the difficulties described. Both the lung function and the radiological changes in the early stages are non-specific, and within this large industrial complex many types of inhaled pollutants can give rise to interstitial fibrosis. Chest radiographic interpretation is subject to observer variability, and we feel it is clearly a major advance to be able, during life, to obtain material for definitive histology. Bearing in mind the obvious hazard of needle lung biopsy, the advantage of a clear-cut diagnosis on histological grounds is of great value and has been welcomed by the Pneumoconiosis Panel and the Industrial Medical Services.

We take this opportunity of thanking Dr. Eric Knowles, of I.C.I. Billingham Division on Teesside, for his interest and assistance, and also Mr. F. C. Jordan, of the Central Clinical Laboratory, for his technical help in processing the biopsy material.

\section{REFERENCES}

Hunt, R. (1965). Routine lung function studies on $830 \mathrm{employees}$ in an asbestos processing factory. Ann. N.Y. Acad. Sci., 132, Art. 1, 406. Northern Daily Mail (1965). "Killer Dust", 6 November 1965.

Smith, W. G. (1964a). Needle biopsy of the lung. Thorax, 19, 68.

- (1964b). Letter. Brit. med. F., 1, 1710.

- (1968). Personal communication.

Thomson, M. L., Pelzer, A., and Smither, W. J. (1965). The discriminant value of pulmonary function tests in asbestosis. Ann. N.Y. Acad. Sci., 132, Art. 1, 421.

Williams, R., and Hugh-Jones, P. (1960a). The radiological diagnosis of asbestosis. Thorax, 15, 103 .

- (1960b). The significance of lung function changes in asbestosis. Ibid.. 15, 109. 